\title{
Physical Activity Prescription for Chronic Diseases: Knowledge of Healthcare Professionals in Hospital Setting
}

\author{
Dr. Getu T.* \\ Department of Sports Science, Debremarkos University, Ethiopia \\ *Corresponding Author: Dr. Getu T., Department of Sports Science, Debremarkos University, Ethiopia
}

\begin{abstract}
:
Introduction: Physical inactivity is a fast-growing public health problem and contributes to a variety of chronic diseases and health complications. In addition to improving a patient's overall health, increasing physical activity has proven effective in the treatment and prevention of chronic diseases.
\end{abstract}

Aim: The aim of this study is to describe the knowledge and attitude of Ethiopian, particularly Addis Ababa's healthcare professionals on exercise prescription.

Methods: A cross-sectional self-administered questionnaire was adapted and developed according to guidelines from literature to assess the knowledge of healthcare professionals' physical activity prescription. The questionnaire was completed by a total of 387 HCPs from 422 sample healthcare professionals in 7 government hospitals in Addis Ababa. The outcomes measures include the demographic information, interest of training, educational preparation regarding exercise prescription, role of healthcare professionals on exercise prescription as well as their Knowledge regarding recommendation and formulation of exercise prescriptions were also assessed.

Results: Respondents prescribe PA for a variety of reasons: promote general wellbeing (24.8\%), treat chronic diseases( 19.3\%), prevent chronic diseases( 17\%), and to control patients weight(38.9\%). The most common methods that the HCPs' used to PPA was discuss with patients about PA (68.2\%). Only 39.3\% of $H C P s$ were received educational preparation to counsel their patients on PA, the area that most commonly received were: health benefits of PA (47.8\%). There was a significant difference about educational preparation in relation to PAP among HCPs. About $95 \%$ of healthcare professionals reported that they would like in obtaining training related to PAP. Regarding knowledge of healthcare professionals to PPA, indicates that low level of knowledge. Perhaps the result of this study indicates that there is a significance difference regarding to knowledge to prescribe PA for their patients among HCPs. Most of healthcare professionals recommend walking type of $P A$ to their patients(42.9\%).

Conclusion: The results of this study indicates that healthcare professionals have insufficient knowledge to prescribe physical activity and they have less preparation about physical activity prescription in their educational program. This study findings show that a need for trainings in related to physical activity prescription/counseling for HCPs.

Keywords: Physical Activity Prescription, Healthcare Professionals, Educational Preparation and Knowledge

\section{INTRODUCTION}

Physical inactivity is a fast-growing public health problem and contributes to a variety of chronic diseases and health complications, including obesity, diabetes and cancer. The human body needs frequent physical activity to function properly (Hallal PC;2012). Doctors are well positioned to provide physical activity (PA) counseling to patients. They are a respected source of health-related information and can provide continuing preventive counseling feedback and follow-up; they may have ethical obligations to prescribe PA (Lobelo F., Duperly J. and Frank E., 2008). There is evidence for the benefit of exercise in many forms of disease. It is effective, inexpensive, with a low side-effect profile, and can have a positive environmental impact. Despite this, there remains a reluctance within the medical profession to use exercise as a treatment. This probably reflects a lack of knowledge among doctors of the benefits of exercise, and a lack of practical skills in the prescription of exercise for persons with chronic disease. Frequently, the risks of exercise are 
misunderstood and overestimated ( Royal College of Physicians, 2012). The role of health care providers ( nurses, physicians, physiotherapist etc.) have great value for promoting physical activity in hospitals for prevention and treatment of chronic disease. But some studies show that they are not doing match on physical activity prescription and counseling ( $B M J, 2015)$.

Regular PA plays an important role in the primary and secondary prevention of several chronic diseases. The WHO has identified physical inactivity as the fourth leading risk factor for global mortality. Physical inactivity levels are rising in many countries, with major implications for the prevalence of NCDs and the general health of the population worldwide. Global recommendations on PA for health are formulated as primary prevention of NCDs through PA at population level (WHO 2010, ACSM 2009). The significance of physical activity on public health, the global mandates for the work carried out by WHO in relation to promotion of physical activity and NCDs prevention, and the limited existence of national guidelines on physical activity for health in low- and middle-income countries (LMIC) make evident the need for the development of global recommendations that address the links between the frequency, duration, intensity, type and total amount of physical activity needed for the prevention of NCDs. The focus of the Global Recommendations on Physical Activity for Health is primary prevention of NCDs through physical activity at population level, and the primary target audience for these Recommendations are policy-makers at national level ( WHO, 2010). Physical inactivity(PI) has been identified as the fourth leading risk factor for global mortality $(6 \%$ of deaths globally). This follows high blood pressure (13\%), tobacco use (9\%) and high blood glucose (6\%). Overweight and obesity are responsible for 5\% of global mortality (WHO, 2009). The link between physical inactivity and CHD is well recognized. The World Health Report estimated that over 20\% of CHD in developed countries is due to lack of physical activity (WHO, 2002).

There is evidence for the benefit of exercise in many forms of disease. Despite this, there remains a reluctance within the medical profession to use exercise as a treatment. This probably reflects a lack of knowledge among doctors of the benefits of exercise, and a lack of practical skills in the prescription of exercise for persons with chronic disease. Frequently, the risks of exercise are misunderstood and overestimated ( Royal College of Physicians, 2012). Research shows that health care providers who themselves act on the advice they give provide better counseling and motivation of their patients to adopt such health advice. But when we see the actual practice of health care providers regarding to prescribe/counseling PA for patients shows low. For example, rates of exercise counseling by doctors remain low; only $34 \%$ of US adults report exercise counseling at their last medical visit (Lobelo F., Duperly J. and Frank E, 2009). Participants appeared to have a positive attitude to PAP but reported a low level of knowledge about benefits of PAP and how PAP should be used (Svensson S.,2012). Medical schools need to increase the proportion of students adopting and maintaining regular PA habits to increase the rates and quality of future PA counseling delivered by physicians (Milton K, Smith B, Bull F, 2014)

\subsection{Aim of Study}

The primary aim of this study was to describe the knowledge of Ethiopian healthcare professionals pertaining to exercise prescription.

\subsection{Research Objectives}

- Examining educational training related to PA prescription/counseling among healthcare professionals participating in a cross-sectional study.

- Describing self-perceived knowledge of healthcare professionals participating in a cross-sectional study toward prescribe/counsel PA.

\subsection{Research Hypothesis}

- There will be no significant differences among healthcare professionals participating in a crosssectional study in self-perceived knowledge to counsel PA.

- There will be no significant differences in the educational preparation related to PA counseling among healthcare professionals participating in a cross-sectional study. 


\section{Method}

A cross-sectional survey design was used to assess Addis Ababa's healthcare professionals' knowledge about physical activity counseling and prescription behavior for chronic (noncommunicable) diseases in hospital setting. Descriptive research includes surveys and fact-finding enquiries of different kinds. The major purpose of descriptive research is to describe the state of affairs as it exists at present (Kothari. C.K., 2004).

Addis Ababa city would provided the setting for the study. Seven sample hospitals were selected randomly based on the inclusion and exclusion criteria from 12 hospitals.. Sample Size: The sample size was determined by using the formula for estimating a single population proportion. Sample size was calculated by taking the proportion of physical activity prescription/counseling which is $50 \%$ on healthcare professionals (medical doctors, nurses and physiotherapist) for chronic disease with 95\% confidence level, 5\% margin of error to get an optimum sample size. Based on the above assumptions, the formula is as follows:

$\mathrm{s}=\mathrm{X} 2 \mathrm{NP}(1-\mathrm{P}) \div \mathrm{d} 2(\mathrm{~N}-1)+\mathrm{X} 2 \mathrm{P}(1-\mathrm{P})$

$\mathrm{s}=$ required sample size

$\mathrm{X} 2=$ the table value of chi-square for 1 degree of freedom at the desired confidence level $1.96 \times 1.96=3.8416$

$\mathrm{N}=$ the population size

$\mathrm{P}=$ the population proportion (assumed to be.50 since this would provide the maximum sample size).

$\mathrm{d}=$ the degree of accuracy expressed as a proportion (.05). Based on this formula the sample would be 384 , assume $85 \%$ will be return rate, then add $15 \%$, the total sample will be 442 .

\subsection{Measures and Data Collection Instruments}

Healthcare professional's knowledge about prescribing/counseling physical activity for their patients. Healthcare professional's knowledge will measure by two types of questionnaires: a) the first 5 items' answer is anchored on a five-point scale ranging from Poor/no knowledge $=1$ to Excellent=5: 1) current physical activity recommendations to advise patients, 2) about different physical activity intensities (light, moderate, vigorous), 3) knowledge about assessing clients for physical activity, 4) knowledge about the effects of physical activity on chronic diseases, 5) knowledge on prescribing specific physical activity programs for patients. From these the first $1 \& 2$ items adapted from Galaviz et al., (2014) and ACSM (2009, 2004) with some modifications and item 3 \& 4 adapted from promotion of physical activity by health practitioner's questionnaire (PPAHQ). It is a modified version of the Promotion of Physical Activity by Nurse Practitioners Questionnaire (Buchholz \& Purath, 2007; Burns et al., 2000; as cited in Grimstvedt; 2011). Item 5 added by the researcher. And b) the second 5 items are: what method(s) do you use to determine a client's physical activity level, the principles of physical activity/exercise prescription, method do you use to provide physical activity counseling for your clients, and reasons for counseling physical activity (verbal/ written prescription) for your patients. Answering with rank by numbering the one you most frequently use as 1 , second most frequently use as 2 etc. from the given choice, adapted from promotion of physical activity by health practitioner's questionnaire (PPAHQ), (Grimstvedt; 2011).

The data analyses were conducted with the Statistical Package for Social Sciences (SPSS version 20 ). Simple descriptive statistics, One-way ANOVA and correlation were used to examine associations and differences among variables

\section{RESUltS}

Knowledge of healthcare professionals about physical activity prescription/counseling were assessed using five self reported questions. The participants were asked to indicate their knowledge a 5-point scale were $1=$ no/poor knowledge and 5 very good knowledge (see Table 3.1). The results of this study indicates that the participants perceived themselves as at least having good knowledge $(19.8 \%$ $=$ good + very good knowledge) on current physical activity recommendations, (22.6\%) about different PA intensities (light, moderate \& vigorous), Knowledge about assessing clients for physical 
activity (20.2\%), knowledge about the effect of physical activity on non-communicable diseases (41.6\%) and healthcare professionals had relatively least perceived knowledge on prescribed specific physical activity to patients (13.4\%).

Male healthcare professionals had statistically greater knowledge about physical activity prescription/counseling: the effects of physical activity on non-communicable diseases $(\mathrm{M}=2.59$ and 1.91, male, female respectively), on current physical activity recommendations ( $M=2.14$ and 1.75), about different PA intensities (light, moderate \& vigorous $(\mathrm{M}=2.23$ and 1.79), assessing clients for PA ( $\mathrm{M}=173, \mathrm{M}=2.11)$, effect of PA on NCDs $(\mathrm{M}=2.2, \mathrm{M}=2.6)$ and knowledge on prescribing specific PA( $\mathrm{M}=1.45, \mathrm{M}=1.89)$. In general male healthcare professionals were had more knowledge than female healthcare professionals to prescribe/counseling PA.

Table3.1. Knowledge of healthcare professionals on PAP

\begin{tabular}{|l|l|l|l|l|l|}
\hline \multicolumn{1}{|c|}{ Items } & Poor $(\boldsymbol{\%})$ & Fair $(\boldsymbol{\%})$ & Good $(\boldsymbol{\%})$ & Very good $(\boldsymbol{\%})$ & Mean \\
\hline Current PA recommendation(n= 379) & 34.3 & 45.9 & 12.1 & 7.7 & 1.93 \\
\hline PA intensities (n= 381) & 34.1 & 43.3 & 11.8 & 10.8 & 1.99 \\
\hline Assessing clients for PA & 37.2 & 42.6 & 12 & 8.2 & 1.91 \\
\hline Effect of PA on NCDs & 15.9 & 42.6 & 28.5 & 13.1 & 2.39 \\
\hline Prescribing specific PA & 52.2 & 34.5 & 8.7 & 4.7 & 1.65 \\
\hline
\end{tabular}

Note: $\mathrm{P}=$ Poor/ No knowledge, $\mathrm{F}=$ Fair knowledge, $\mathrm{G}=$ Good Knowledge, $\mathrm{VG}=$ Very good knowledge

Exercise prescription should include the intensity, duration, type and frequency of exercise session (FITT). These FITT principles are very common foundational knowledge in exercise prescription and should be familiar to healthcare professionals to prescribe physical activity for their patients. In this study to examine the intensity of exercise recommended by HCPs, ask the participants to rank by numbering the one you most frequently use as 1 from the listed intensity of physical activity(see Table 3.2). The result shows that most of HCPs $(70 \%)$ were not used specified intensity of physical activity, the second most recommended intensity of physical activity was moderate intensity of a brisk walking 3-4 kmph $(50.1 \%)$ and the third most recommended exercise was intensity $124-143 \mathrm{bpm}$ of heart rate $(43.5 \%)$.

Type of physical activity recommended by HCPs was examined by the question: rank by numbering the one you most frequently use as 1 from the listed type of physical activity (see Table 2). the result of this study shows that walking was the first most commonly prescribed type of physical activity by healthcare professionals (43\%), the second most recommended PA was jogging (32.5\%) and the third most prescribed type of physical activity was aerobic class activity $(27.7 \%)$. Regarding to frequency and duration of pysical activity prescription, (32.6\%) and (38.7\%) of healthcare professionals report that the first most and third most recommonded frequency of physical activity was three days per week respectively, the second most recommonded frequency of exercise prescription was two days per week $(39.7 \%)$. The first most commonly reported duration of physical activity prescription was a total of 30 minute per day $(67.2 \%)$, and a total of 20 minute per day $(54.2 \%)$ and 45 minutes per day $(66.9 \%)$ were second and third most recommonded duration of physical activity prescription respectively.

Reasons of healthcare professionals to prescribe /counsel physical activity for their patients are different. Participants who prescribed/ counseled physical activity to their patients were presented with four questions related to the specific purposes of their prescription/counseling (tick all that apply): promote general wellbeing, treat chronic diseases, prevent chronic diseases and control patients weight (see Table 3.3 ). About (24.8\%) of healthcare professionals were prescribe/counsel physical activity to their patients for the purpose of promoting general wellbeing, for the purpose of treating non-communicable diseases(19.3\%), for the purpose of preventing non-communicable diseases and most of healthcare professionals (38.9\%) were prescribe/counsel PA for the purpose of controlling patients weight. The most commonly identified methods of physical activity counseling/prescription as ranked by participants are provided in Table 3. Discussing physical activity with patients was the most common methods of physical activity counseling $(68.2 \%)$, followed by refer patient to exercise specialist $(25 \%)$. 
Physical Activity Prescription for Chronic Diseases: Knowledge of Healthcare Professionals in Hospital Setting

Table3.2. Knowledge and practice of healthcare professionals on components of physical activity prescription.

\begin{tabular}{|c|c|c|c|}
\hline Types of physical activity $^{\text {a }}$ & $\begin{array}{lr}\text { Percentage } & \text { of } \\
\text { participants } & \text { making } \\
\text { types of } & \text { PAP } \\
\text { components } & \text { as their } \\
\# 1 \text { practice } & \\
\end{array}$ & $\begin{array}{lr}\text { Percentage } & \text { of } \\
\text { participants } & \text { making } \\
\text { types of } & \text { PAP } \\
\text { components } & \text { as their } \\
\# 2 \text { practice } & \\
\end{array}$ & $\begin{array}{lr}\text { Percentage } & \text { of } \\
\text { participants } & \text { making } \\
\text { types of } & \text { PAP } \\
\text { components as their } \\
\# 3 \text { practice }\end{array}$ \\
\hline Walking & $43.0 \%$ & $18.1 \%$ & $7.9 \%$ \\
\hline Jogging & $11.9 \%$ & $32.5 \%$ & $11.6 \%$ \\
\hline Biking/cycling & $3.8 \%$ & $4.6 \%$ & $15.5 \%$ \\
\hline Swimming & $0.35 \%$ & $4.4 \%$ & $1.5 \%$ \\
\hline Aerobics class & $15.2 \%$ & $16.5 \%$ & $27.7 \%$ \\
\hline Home based activities & $15.7 \%$ & $11.2 \%$ & $14 \%$ \\
\hline Strength exercise & $2.7 \%$ & $8.5 \%$ & $13.4 \%$ \\
\hline Stretching exercise & $7.4 \%$ & $4.2 \%$ & $8.2 \%$ \\
\hline \multicolumn{4}{|l|}{ Intensity of $\mathbf{P A}{ }^{\mathbf{a}}$} \\
\hline $60-85 \%$ of their MHR & $7.6 \%$ & $11.6 \%$ & $7.6 \%$ \\
\hline 95-124 bpm of HR & $8.7 \%$ & $16.5 \%$ & $11 \%$ \\
\hline $124-143 \mathrm{bpm}$ of HR & $3.1 \%$ & $3.6 \%$ & $43.5 \%$ \\
\hline $143-162 \mathrm{bpm}$ of HR & $4.1 \%$ & $10.2 \%$ & $14.6 \%$ \\
\hline 300 kilocalorie expenditure/session & $3.5 \%$ & $4.7 \%$ & $19.5 \%$ \\
\hline $\begin{array}{l}\text { Moderate intensity of a brisk } \\
\text { walking 3-4 kmph }\end{array}$ & $2.9 \%$ & $50.1 \%$ & $3.5 \%$ \\
\hline Not specified & $70 \%$ & $3.4 \%$ & $.3 \%$ \\
\hline \multicolumn{4}{|l|}{ Frequency of PAP } \\
\hline Once/day & $26.7 \%$ & $10.0 \%$ & $14.4 \%$ \\
\hline Two days/week & $29.4 \%$ & $39.7 \%$ & $8.0 \%$ \\
\hline Three days/week & $32.6 \%$ & $28.5 \%$ & $38.7 \%$ \\
\hline Five days/week & $11.4 \%$ & $21.8 \%$ & $29.0 \%$ \\
\hline \multicolumn{4}{|l|}{ Duration of PAP } \\
\hline a total of 20 minutes/ day & $15.5 \%$ & $54.2 \%$ & $31.4 \%$ \\
\hline a total of 30 minutes/ day & $67.2 \%$ & $28.3 \%$ & $1.7 \%$ \\
\hline a total of 45 minutes /day & $17.3 \%$ & $17.5 \%$ & $66.9 \%$ \\
\hline
\end{tabular}

$\mathrm{N}=372$

${ }^{a}$ Participants could report more than one type of physical activity prescription component Participants were asked to rank their use of these methods in practice, where 1 indicates the most commonly used. If participants did not use a particular practice, they were asked to leave it blank.

b Percentages will not add up to $100 \%$

Personal physical activity levels was significantly and positively correlated with knowledge of HCPs to prescribe/counsel PA $\left(\mathrm{r}_{\mathrm{s}}=.306, \mathrm{p}=.000\right)$. This indicates that, the more physically active the participants , they were had a greater knowledge to prescribe/counsel about physical activity for their patients.

\section{DISCUSSION}

Regarding to the type of physical activity recommended by healthcare professionals to their patients, type of physical activity recommended by HCPs was examined by the question: rank by numbering the one you most frequently use as 1 from the listed type of physical activity (see Table 4.4.2). the result of this study shows that walking was the first most commonly prescribed type of physical activity by healthcare professionals (43\%), the second most recommended PA was jogging $(32.5 \%)$ and the third most prescribed type of physical activity was aerobic class activity (27.7\%). And the other like biking/cycling, swimming, home based activities, strength exercise and stretching exercise were not commonly recommended physical activities. Similarly in one research conducted on practice patterns, counseling and promotion of physical activity by sports medicine physicians (Pojednic, et al 2016) revealed that the most recommended physical activity for patients by healthcare professionals, asked to what percentage of patients the physicians recommended each activity, the results include: walking $(75 \%)$, aerobic sports $(60 \%)$, strength training $(51 \%)$, cycling $(44 \%)$, swimming $(36 \%)$, yoga $(27 \%)$, team sports $(19 \%)$ and dancing $(13 \%)$. 
Half of the doctors $(51.4 \%)$ were familiar with the WHO recommendation for PA for adults. Nearly one-fourth of the doctors $(24.7 \%)$ reported that they had attended classes/sessions on PA in medical college and $26 \%$ reported that they received formal training for PA counseling. A large majority of the doctors $(78 \%)$ agreed that there is a role for PA in primary and secondary $(91.1 \%)$ prevention of chronic diseases. All the participants agreed that PA has a beneficial role in the prevention of heart diseases, obesity and diabetes. Over half of them (52\%) reported the beneficial role of PA in depression, $22.6 \%$ in chronic respiratory disease and $19.8 \%$ in cancers. Eighty-one per cent of doctors perceived the importance of exercise apart from daily work-related activities. However, only 34.9\% reported that they made a conscious effort to perform regular exercise (Patra, Mini, Mathews, et al, 2015 ).

The implementation of medical school interventions to increase number of students who have good PA habits should constitute a powerful strategy to increase the rates and quality of future healthcare professionals provide PA counseling/prescription. Subsequently, this could have a large impact on the management and prevention of chronic diseases in both developed and developing countries (Lobelo F., Duperly J. and Frank E., 2009). Physicians' health matters and their personal PA practices affect their clinical PA attitudes and physical activity counseling/prescription practices. This relationship is strong and independent of many demographic variables, training and clinical practice factors (Lobelo F., Duperly J. and Frank E., 2009).

\section{CONCLUSION}

To prescribe/counsel physical activity for patients required knowledge on physical activity. Hence healthcare professionals should have sufficient knowledge on principles of physical activity prescription, the effect of PA and current recommendations about PA. The results of this study revealed that healthcare professionals had relatively poor knowledge to prescribe/counsel physical activity specially specific physical activity prescription for patients. In fact, various research findings suggested that healthcare professionals should have an adequate basic knowledge to prescribe or counsel a safe and effective physical activity prescription or counseling to their patients in healthcare setting on their routine practices.

Most of healthcare professionals were not use physical activity prescription principles properly in their prescription/counseling practices. Physical activity prescription components/ principles are fundamental to physical activity recommendation imply that healthcare professionals were not prescribe physical activity in a clear way by including different type of physical activity, frequency, duration, intensity and restrictions. The FITT principles are very common foundational knowledge in exercise prescription and should be familiar to healthcare professionals to prescribe physical activity for their patients. The results have shown that most of HCPs were not used specified intensity of physical activity, the second most recommended intensity of physical activity was moderate intensity of a brisk walking and the third most recommended exercise was intensity of 124-143 bpm of heart rate. The present study revealed that the most commonly identified methods of physical activity counseling/prescription was discussing physical activity with patients. There were significance differences among the three group of healthcare professionals with regard knowledge toward physical activity prescription/counseling and healthcare professionals PAL.

\section{REFERENCES}

[1] Abramson S, Stein J, Schaufele M, Frates E, and Rogan S.( 2000). Personal exercise habits and counseling practices of primary care physicians: a national survey. Clin J Sport Med. Jan;10(1):40-8.

[2] American College of Sports Medicine (2009). ACSM's guidelines for Exercise Testing and Prescription (8th ed), Philadelphia, PA: Lippincott Williams \& Wilkins

[3] BMJ (2015). Exercise: not a miracle cure, just good medicine. $B M J ; 350: \mathrm{h} 1416$

[4] Hallal PC, Andersen LB, Bull FC, Guthold R, Haskell W, Ekelund U.( 2012). Global physical activity levels: surveillance progress, pitfalls, and prospects. The Lancet,380(9838):247-257.

[5] Lobelo F., Duperly J. and Frank E.( 2009). Physical activity habits of doctors and medical students influence their counseling practices, Br J Sports Med 2009;43:89-92. doi:10.1136/bjsm.2008.055426

[6] Milton K, Smith B, Bull F. (2014). Action area 3: Health care. In: Blueprint for an active Australia (2nd ed). Melbourne: National Heart Foundation of Australia. 
[7] Patra L., Mini G.K., Mathews E., and Thankappan K.R.(2015). Doctors' self-reported physical activity, their counseling practices and their correlates in urban Trivandrum, South India: should a full-service doctor be a physically active doctor?, Br J Sports Med;49: 413-41

[8] Pojednic RM., Polak R., Arnstein F., Kennedy MA., Amy Bantham A. and Phillips BA.(2016). Practice patterns, counseling and promotion of physical activity by sports medicine physicians,. JSci Med Sport,

[9] Ribera A. P.,McKenna J., Riddoch C. (2005). Attitudes and practices of physicians and nurses regarding physical activity promotion in the Catalan primary health-care system. European Journal of Public Health: 15(6), 569-575

[10] Royal College of Physicians (2012): Exercise for life: physical activity in health and disease. London: RCP.

[11] WHO (2002). The World Health Report 2002: Reducing Risks, Promoting Healthy Life, Geneva: World Health Organization

[12] WHO (2010a). Global Status Report on Noncommunicable Diseases. Geneva; Switzerland: WHO.

Citation: Dr. Getu T.. " Physical Activity Prescription for Chronic Diseases: Knowledge of Healthcare Professionals in Hospital Setting" International Journal of Sports and Physical Education (IJSPE), vol 4, no. 2, 2018, pp. 1-7. doi:http://dx.doi.org/10.20431/2454-6380.0402001.

Copyright: (C) 2018 Authors. This is an open-access article distributed under the terms of the Creative Commons Attribution License, which permits unrestricted use, distribution, and reproduction in any medium, provided the original author and source are credited. 